\title{
newly on the market
}

These descriptions are prepared by the staff of Nature on the basis of material provided by manufacturers. There is a Reader Enquiry Form facing page 28.

$25 \mathrm{MHz}$ oscilloscope. Pye Unicam. A $25 \mathrm{MHz}$ oscilloscope with full digital measuring facilities found normally only on higher frequency models, the Philips PM 3214 has all the features of a general purposes oscilloscope with high $2 \mathrm{mV}$ sensitivity over the full bandwidth. Digital measurements are facilitated by such features as a delayed timebase with fully calibrated delay and an alternate timebase facility which allows display of both main and delayed timebase signals at the same time for direct and easy reference. The wide range of power supply possibilities allows the oscilloscope to operate in the laboratory or the field. Mains supplies of $110,127,220$ and $240 \mathrm{~V}$ a.c. $\pm 10 \%$ at $46-440 \mathrm{~Hz}, 21-27 \mathrm{~V}$ d.c. supplies or an internal $24 \mathrm{~V}$ battery option are the alternatives. Power consumption of the oscilloscope is $30 \mathrm{~W}$.

Circle No. 42 on Reader Enquiry Form

Fluorescence detector. Perkin-Elmer Ltd. A new fluorescence detector for liquid chromatography, LC-1000, has been designed with emphasis on high sensitivity, selectivity and ease of operation. The LC-1000 uses a xenon source providing a continuum over the $\mathrm{UV} /$ visible region. The square section quartz flow cell of $20 \mu \mathrm{l}$ volume is designed to provide maximum sensitivity, minimum scatter and no detectable loss of peak resolution while still being simple to dismantle for cleaning. Model LC-1000 uses a continuous interference filter as an emission monochromator operating over the region $390-750 \mathrm{~nm}$, and the ability to scan an emission spectrum of a peak in a chromatogram can provide peak identification-a unique advantage. For wavelengths below $390 \mathrm{~nm}$ the fluorescence can be monitored by any fixed interference filter.

Circle No. 43 on Reader Enquiry Form

Photomultiplier power supply. EMI. The Type PM28A has the following characteristics from the electrical specification: output voltages from 100 to $2,800 \mathrm{~V}$, ripple better than $2 \mathrm{mV}$ peak-to-peak, temperature coefficient less than 50 p.p.m. per ${ }^{\circ} \mathrm{C}$ and load regulation 10 p.p.m. from a no load to full load ( $5 \mathrm{~mA})$ change. An additional feature is that the unit can be reset to within $0.2 \mathrm{~V}$ throughout the voltage range so that photomultiplier tube gains can be reproduced accurately. The unit is capable of positive or negative output, with options for remote programming and kits are available to mount units either singly or two abreast into a 19-inch rack.

Circle No. 44 on Reader Enquiry Form

Multiple sample harvesters. Ilacon Ltd. Designed for use in lymphocyte transformation assays, and a wide range of other isotope assays carried out in microplates, these harvesters introduce two unique design features which ensure trouble free and efficient operation. The basic model uses a patent disk cutter system to cut and hold the glass fibre filter paper, and a wash trough which allows complete versatility in washing without the need for switches or valves. Alternative models allow either supernatant collection or a disk clamping system for harvesting on to intact sheets of filter paper.

Circle No. 45 on Reader Enquiry Form

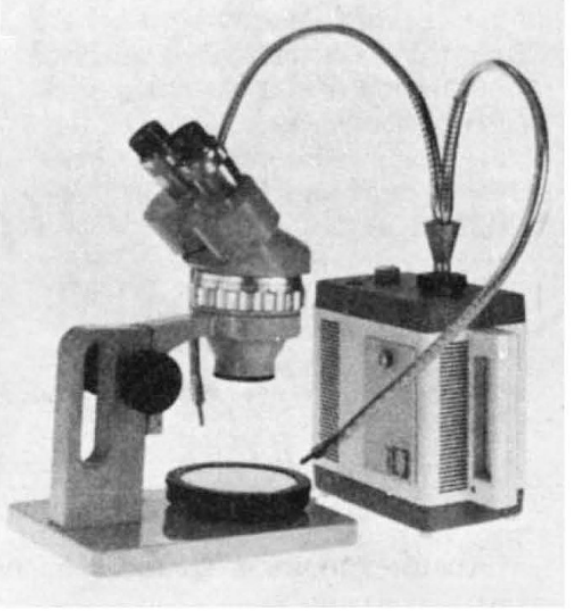

Will Z-33 zoom stereomicroscope.

Will Z-33 zoom stereomicroscope. Will-Strübin. The Will-Strübin stereo zoom microscope has a magnification range from $\times 2.7$ to $\times 180$. The eyepieces give extra wide field vision, and the eyepiece sleeves are adjustable and inclined $45^{\circ}$ which guarantees comfortable vision in the normal sitting position. Various accessories are available including transmitted light universal stands, camera attachments, eyepieces $\times 5, \times 10, \times 15$, and $\times 20$, also auxiliary lenses and fibre optics.

Circle No. 46 on Reader Enquiry Form
Multipoint thermal printing recorder. International Instruments Ltd. The Speed Servo II combines a linear servo motor with a simple solid state semiconductor printing device that ensures positive multipoint channel identification. The printhead thermally brands the three basic types of readout: the channel number and a dot, all dots, or mixed dots and numbers as programmed. Models with up to 24 independent channels are available and the print rate can be as fast as one second per point full scale. The channel identification numbering system automatically prevents confusion when input values cause the traces to overlap, especially when all channels are in use, saving time in chart reading and analysis.

Circle No. 47 on Reader Enquiry Form

Liquid chromatographs. Perkin-Elmer. Series $2 / 1$ is a single module providing both solvent delivery and sample injection and features high pressure capability, rapid solvent changeover, unlimited solvent supply, precise and steady flow, and a wide flow range adjustable from $0.1 \mathrm{ml} \mathrm{min} \mathrm{mi}^{-1}$ to $29 \mathrm{ml}$ $\min ^{-1}$ for analytical and preparative separations. Series $2 / 2$ combines the operating features of two Series $2 / 1$ pumps and a sample injection system. The instrument provides linear solvent and flow programming capability from $0.1 \mathrm{ml} \mathrm{min}{ }^{-1}$ to $30 \mathrm{ml} \mathrm{min}{ }^{-1}$. Series $2 / 2$ also offers direct mixing of selected solvent compositions, preparative flow rates, and allows independent solvent changeover.

Circle No. 48 on Reader Enquiry Form

Bench-top chemostat. New Brunswick Scientific Co., Inc. This accurately defines growth parameters such as agitation, aeration, temperature, nutrient addition and harvesting of cells grown in pure culture. The BioFlo can also be equipped with miniature probes and controllers for dissolved oxygen and $p \mathrm{H}$ regulation. With a working volume of only $350 \mathrm{ml}$, it is possible to sustain cell growth for a minimum of $38 \mathrm{~h}$ without replenishing the medium. The BioFlo can be operated at temperatures from below ambient to $60^{\circ} \mathrm{C}$. A magnetically coupled agitator produces a homogeneous dispersion of culture medium under aseptic conditions at speeds from 200 to 1,000 r.p.m.

Circle No. 49 on Reader Enquiry Form 